\title{
Análisis de indicadores de la calidad asistencial sanitaria del Centro de Salud "A" Fuerte Militar Marco Aurelio Subía, en el periodo comprendido entre julio y diciembre del 2018. @()@(
}

Analysis of indicators of the health care quality of the Health Center "A" Marco Aurelio Subia Military Fort, in the period between july and december of 2018.

\author{
Mayra Jacqueline Zabala Chico ${ }^{1}$, Víctor Manuel Arévalo Méndez $^{2} \&$ Paulina Verónica \\ Cáceres Manzano ${ }^{3}$
}

Recibido: 10-02-2019 / Revisado: 15-02-209 /Aceptado: 04-03-2019/ Publicado: 27-04-2019

\begin{abstract}
.
DOI: https://doi.org/10.33262/cienciadigital.v3i2.1.438

The analysis is performed in a sample of 100 clinical records of patients treated at the "A" Fortress Marco Aurelio Subía Health Center, in the period between July and December 2018 to assess the quality of the records included in said stories, with the purpose of analyzing the indicators of the quality of health care in the first level of attention, and to promote the continuous improvement of the quality in the Health Center "A" FM MAS.

100 clinical records of care registered in the outpatient clinic were included in the period between July and December of 2018, in each one 5 parameters were rated with a total of 1000 points, taking as a reference the model proposed by Ing. Luis Eduardo Pavón and Dr. María Angélica García in the book The Medical Audit as a Control Tool in the Hospital Administration in Ecuador 2017, obtaining as a result that 11 medical records reached a score between $72 \%$ and $78 \%$, equivalent to GOOD, and 89 medical records reached a score between $66 \%$ and $70 \%$ equivalent to BAD.
\end{abstract}

Keywords: Analysis, indicators, quality, assistance, health, health

\footnotetext{
${ }^{1}$ Universidad Nacional de Chimborazo. Riobamba. Ecuador mjzabach@ hotmail.com

${ }^{2}$ Universidad Nacional de Chimborazo. Riobamba. Ecuador pepito53@ hotmail.com. 


\section{Resumen.}

Se realiza el análisis en una muestra de 100 historias clínicas de pacientes atendidos en el Centro de Salud "A" Fuerte Militar Marco Aurelio Subía, en el periodo entre julio y diciembre del 2018 para valorar la calidad de los registros que se incluyen en dichas historias, con la finalidad de analizar los indicadores de la calidad de atención sanitaria en el primer nivel de atención, e impulsar la mejora continua de la calidad en el Centro de Salud "A” FM MAS.

Se incluyeron 100 Historias clínicas de atenciones registradas en la consulta externa en el periodo comprendido entre julio y diciembre del 2018, en cada una se calificó 5 parámetros con un total 1000 puntos, tomando como referencia el modelo propuesto por el Ing. Luis Eduardo Pavón y Dra. María Angélica García en el libro La Auditoria Médica como Herramienta de Control en la Administración Hospitalaria en el Ecuador 2017, obteniéndose como resultado que 11 historias clínicas alcanzaron un puntaje entre $72 \%$ y $78 \%$, equivalente a BUENO, y 89 historias clínicas alcanzaron un puntaje entre $66 \%$ y $70 \%$ equivalente a MALO.

Palabras claves: Análisis, indicadores, calidad, asistencial, sanitaria, salud

\section{Introducción.}

Al tener una objeción del 8 al 10\% en el planillaje y facturación por parte del ISSFA en las atenciones realizadas en el C.S "A" FM MAS, es necesario realizar un análisis de la relación existente entre dichas objeciones con la calidad del llenado de la historia clínica, así como determinar cuál es el parámetro con mayor falla en el registro.

La preocupación por atender a los pacientes con la mayor calidad posible es tan antigua como la práctica médica, haciéndose énfasis en la importancia de la calidad en la asistencia sanitaria. Entendiéndose calidad de salud como el grado de aproximación entre lo que el usuario espera, en función de sus expectativas y necesidades y la atención que recibe, en función del conocimiento, tecnología y recursos disponibles. ${ }^{1}$

La gestión de la calidad de todas las organizaciones, sin importar su tipo, tiene un papel determinante la normalización o regulación a partir de normas dirigidas a establecer soluciones estandarizadas ante situaciones repetitivas.

Con el conocimiento de los problemas de calidad y seguridad obtenida a partir de indicadores, estudios epidemiológicos se puede realizar estrategias y planes de mejora. Del mismo modo como el conocimiento de los problemas es importante también es fundamental es el conocimiento de la sostenibilidad de las posibles soluciones y la inspección, seguimiento y control de la implementación de las actuaciones.

La Constitución de la República del Ecuador de 2008 señala que "la salud es un derecho que garantiza el Estado"2, teniendo como ente rector al Ministerio de Salud Pública encargado de normar, regular y controlar las actividades realizadas por entidades públicas y privadas que se vinculan con la salud de las personas y el medio ambiente ${ }^{3}$, el mismo que como parte del fortalecimiento de la salud y como mandato constitucional establece "La red pública integral de salud será parte del Sistema Nacional de Salud y estará conformada por el 
conjunto articulado de establecimientos estatales, de la seguridad social y con otros proveedores que pertenecen al Estado, con vínculos jurídicos, operativos y de complementariedad" ${ }^{4}$.

Las unidades de salud de la Fuerza Terrestre como parte de la Red Pública de Salud brindan servicios de salud en todos los niveles, tratando de que sean de manera oportuna y con calidad a los ecuatorianos, aunque en el subsistema de salud aún no se establecen las Unidades de Gestión de Calidad y con las limitaciones en la mejora continua y la calidad de los servicios de salud.

La Organización Mundial de la Salud define la calidad asistencial de la siguiente manera: Una atención sanitaria de alta calidad es la que identifica las necesidades de salud (educativas, preventivas, protectoras y de mantenimiento) de los individuos o de la población de una forma total y precisa, y destina los recursos necesarios (humanos y de otros tipos) a estas necesidades de manera oportuna y tan efectiva ${ }^{5}$.

De esta definición se derivan tres características importantes que engloba la Calidad asistencial: el conocimiento científico y actualizado que debe poseer el personal de salud, los recursos materiales y humanos siendo fundamental contar con los recursos necesarios para poder ofrecer servicios de calidad y comprender la problemática de salud de una forma global que abarca desde el conjunto de necesidades hasta educación.

La historia clínica es una fuente valiosa de información privada del paciente sobre la actividad médica desarrollada en él ${ }^{6}$, siendo el documento obligatorio en el acto médico sea privado, público (Hospitalario o en Atención Primaria de Salud) para garantizar la calidad asistencial y la continuidad en la atención ${ }^{7}$. Al mismo tiempo sirve como un instrumento de inicio de estudios de investigación, mediante los cuales se pueden valorar las necesidades prioritarias y establecer los procedimientos, estrategias implementadas y resultados obtenidos por el equipo de salud ${ }^{8}$.

\section{Metodologia.}

Se tomó una muestra de 100 historias clínicas de manera aleatoria, correspondientes al 8\% de las atenciones registradas en el período comprendido entre julio y diciembre del 2018 para análisis de la calidad en el llenado de las historias clínicas del Centro de Salud "A" Fuerte Militar Marco Aurelio Subía, perteneciente a la Brigada de Aviación del Ejército, en el mismo que se atiende, personal militar, familiares, derechohabientes y civiles de la zona de influencia.

La muestra se obtuvo en relación al 8 a $10 \%$ de objeciones recibidas en el proceso de facturación de las atenciones registradas en historias clínicas en el período en estudio.

Los parámetros que se evaluaron en las historias clínicas del área de consulta externa son:

1. Calidad de registro en la historia clínica

2. Metodología diagnóstica

3. Notas de evolución 
4. Tratamiento y ordenes médicas

5. Evaluación final - observaciones

\section{Resultados.}

Estos cinco parámetros son propuestos por el Ing. Luis Eduardo Pavón y Dra. María Angélica García en el libro La Auditoria Médica como Herramienta de Control en la Administración Hospitalaria en el Ecuador $2017{ }^{9}$, como el modelo de la siguiente tabla.

Tabla $\mathrm{N}^{\circ} 1$. Calidad de registro en la historia clínica

Tomado de: Pavón, L. y García, M. La Auditoria Médica como Herramienta de Control en la Administración Hospitalaria en el Ecuador 2017. Ecuador. 2017.

\begin{tabular}{|c|c|c|c|c|}
\hline $\begin{array}{l}\text { CALIDAD DE REGISTRO EN LA } \\
\text { HISTORIA CLINICA }\end{array}$ & AUS & MED & MAX & $\begin{array}{l}\text { PUNTAJE } \\
\text { IDEAL }\end{array}$ \\
\hline Se anota fecha y hora. & 0 & 10 & 20 & 20 \\
\hline Se registra datos de filiación & 0 & 10 & 20 & 20 \\
\hline Se registra anamnesis y examen clínico. & 0 & 30 & 60 & 60 \\
\hline Firma y sello del médico que la elabora. & 0 & 10 & 20 & 20 \\
\hline $\begin{array}{l}\text { Presentación de la historia (pulcritud, orden, } \\
\text { legibilidad, relato claro sin usar abreviaturas). }\end{array}$ & 0 & 10 & 20 & 20 \\
\hline PUNTAJE MÁXIMO & 0 & 70 & 140 & 140 \\
\hline
\end{tabular}

Tabla $\mathbf{N}^{\circ}$ 2. Metodología Diagnóstica

Tomado de: Pavón, L. y García, M. La Auditoria Médica como Herramienta de Control en la Administración Hospitalaria en el Ecuador 2017. Ecuador. 2017.

\begin{tabular}{l} 
METODOLOGIA DIAGNOSTICA AUS MED MAX $\begin{array}{c}\text { PUNTAJE } \\
\text { IDEAL }\end{array}$ \\
\hline
\end{tabular}

\begin{tabular}{|c|c|c|c|c|}
\hline $\begin{array}{l}\text { Exploracion fisica completa por sistemas y } \\
\text { evaluacion prreferencial congruente con } \\
\text { anamenesis }\end{array}$ & 0 & 40 & 80 & 80 \\
\hline $\begin{array}{l}\text { Criterio de diagnostivo fundamentado en } \\
\text { historia clinica }\end{array}$ & 0 & 0 & 80 & 80 \\
\hline $\begin{array}{l}\text { Exámenes auxiliares pertinentes con historia } \\
\text { clínica y diagnóstico presuntivo. }\end{array}$ & 0 & 40 & 80 & 80 \\
\hline $\begin{array}{l}\text { Número de consultas para llegar al Historia } \\
\text { Clínica y diagnóstico presuntivo diagnóstico } \\
\text { definitivo (no mayor de } 2 \text { consultas) }\end{array}$ & 0 & 30 & 60 & 60 \\
\hline Plan de trabajo completo & 0 & 20 & 40 & 40 \\
\hline PUNTAJE MÁXIMO & & & 340 & 340 \\
\hline
\end{tabular}

Tabla $\mathbf{N}^{\circ} 3$. Notas de evolución 
Tomado de: Pavón, L. y García, M. La Auditoria Médica como Herramienta de Control en la Administración Hospitalaria en el Ecuador 2017. Ecuador. 2017.

\section{NOTAS DE EVOLUCION}

Se anota, interpreta resultados de exámenes auxiliares.

Se anota interconsulta e indicaciones del médico consultante.

Se anota si el paciente está de alta, será referido o contra referido.

Evolución de acuerdo al SOAP.

0

30

60

0

Aparece nombre y firma del médico que hizo la nota de evolución.

AUS MED MAX

$0 \quad 40 \quad 80$

80

$0 \quad 0 \quad 20$

$0 \quad 0 \quad 20$

PUNTAJE MÁXIMO

Tabla $\mathbf{N}^{\circ}$ 4. Tratamiento y órdenes del medico

Tomado de: Pavón, L. y García, M. La Auditoria Médica como Herramienta de Control en la Administración Hospitalaria en el Ecuador 2017. Ecuador. 2017.

\begin{tabular}{lcccc}
\hline $\begin{array}{l}\text { TRATAMIENTO Y ORDENES DEL } \\
\text { MEDICO }\end{array}$ & AUS & MED & MAX & $\begin{array}{c}\text { PUNTAJE } \\
\text { IDEAL }\end{array}$ \\
\hline $\begin{array}{l}\text { Tratamiento médico o procedimiento } \\
\text { de Cirugía menor fueron apropiados } \\
\text { de acuerdo a protocolos. }\end{array}$ & 0 & 40 & 80 & 80 \\
$\begin{array}{l}\text { Se anota medicamentos dosis, vía de } \\
\text { administración, periodicidad y } \\
\text { duración. }\end{array}$ & 0 & $20-40$ & 60 & 60 \\
$\begin{array}{l}\text { Se anota el tipo de dieta de acuerdo a } \\
\text { patología cuando es pertinente. }\end{array}$ & 0 & 0 & 20 & 20 \\
$\begin{array}{l}\text { Presencia o ausencia de } \\
\text { complicaciones. }\end{array}$ & 0 & 0 & 60 & 60 \\
\hline \begin{tabular}{l} 
PUNTAJE MÁxIMO \\
\hline
\end{tabular} & & & $\mathbf{2 2 0}$ & $\mathbf{2 2 0}$ \\
\hline
\end{tabular}


Tabla $\mathbf{N}^{\circ}$ 5. Evaluación final y observaciones.

Tomado de: Pavón, L. y García, M. La Auditoria Médica como Herramienta de Control en la Administración Hospitalaria en el Ecuador 2017. Ecuador. 2017.

EVALUACION FINAL Y OBSERVACIONES
AUS

MED
MAX

PUNTAJE

IDEAL

Los documentos de la Historia

Clínica aparecen en el orden establecido por las normas

$\begin{array}{llll}0 & 10 & 20 & 20\end{array}$

institucionales.

En caso de enfermedades de vigilancia Epidemiológica, obligatoria se comunicó 0 0 10

oportunamente a epidemiología.

Se consignó código alfanumérico (CIE-10)

0

0

20

20

Uso de formatos oficiales en la historia clínica.

10

20

20

En contenido de la Historia

Clínica es útil para la enseñanza

0

0

30

30

e investigación.

PUNTAJE MÁXIMO

100

100

Durante el período entre julio y diciembre del 2018, en los archivos generales del centro de salud constan registradas un total de 1200 consultas en medicina general, de las cuales se evalúan 100 historias clínicas, mediante 5 parámetros de revisión, obteniendo los siguientes resultados:

Tabla 6. Contenido de calidad de registro en la historia clínica.

\begin{tabular}{|c|c|c|c|c|c|c|}
\hline $\begin{array}{l}\text { EVALUACION FINAL Y } \\
\text { OBSERVACIONES }\end{array}$ & AUS & MED & MAX & $\begin{array}{l}\text { PUNTAJE } \\
\text { IDEAL }\end{array}$ & $\begin{array}{c}\text { PUNTAJE } \\
\text { ALCANZADO }\end{array}$ & $\%$ \\
\hline $\begin{array}{l}\text { Los documentos de la Historia } \\
\text { Clínica aparecen en el orden } \\
\text { establecido por las normas } \\
\text { institucionales. }\end{array}$ & 0 & 10 & 20 & 20 & 20 & $14 \%$ \\
\hline $\begin{array}{l}\text { En caso de enfermedades de } \\
\text { vigilancia Epidemiológica, } \\
\text { obligatoria se comunicó } \\
\text { oportunamente a epidemiología. }\end{array}$ & 0 & 10 & 20 & 20 & 20 & $14 \%$ \\
\hline $\begin{array}{l}\text { Se consignó código alfanumérico } \\
\text { (CIE-10) }\end{array}$ & 0 & 30 & 60 & 60 & 33 & $23,60 \%$ \\
\hline $\begin{array}{l}\text { Uso de formatos oficiales en la } \\
\text { historia clínica. }\end{array}$ & 0 & 10 & 20 & 20 & 20 & $14 \%$ \\
\hline $\begin{array}{l}\text { En contenido de la Historia } \\
\text { Clínica es útil para la enseñanza } \\
\text { e investigación. }\end{array}$ & 0 & 10 & 20 & 20 & 19,40 & $13,90 \%$ \\
\hline PUNTAJE MÁXIMO & & & 140 & 140 & 93 & $42.00 \%$ \\
\hline
\end{tabular}


Tabla 7. Contenido de metodología diagnóstica en la historia clínica.

\begin{tabular}{|c|c|c|c|c|c|c|}
\hline METODOLOGIA DIAGNOSTICA & AUS & MED & MAX & $\begin{array}{l}\text { PUNTAJE } \\
\text { IDEAL }\end{array}$ & $\begin{array}{c}\text { PUNTAJE } \\
\text { ALCANZADO }\end{array}$ & $\%$ \\
\hline $\begin{array}{l}\text { Exploración física completa por sistemas y } \\
\text { evaluación preferencial congruente con } \\
\text { anamnesis. }\end{array}$ & 0 & 40 & 80 & 80 & 80 & $23.50 \%$ \\
\hline $\begin{array}{l}\text { Criterio diagnóstico fundamento en historia } \\
\text { clínica }\end{array}$ & 0 & 0 & 80 & 80 & 80 & $23.50 \%$ \\
\hline $\begin{array}{l}\text { Exámenes auxiliares pertinentes con historia } \\
\text { clínica y diagnóstico presuntivo. }\end{array}$ & 0 & 40 & 80 & 80 & 79.2 & $23.30 \%$ \\
\hline $\begin{array}{l}\text { Número de consultas para llegar al diagnóstico } \\
\text { definitivo (no mayor de } 2 \text { consultas) }\end{array}$ & 0 & 30 & 60 & 60 & 60 & $17.60 \%$ \\
\hline Plan de trabajo completo & 0 & 20 & 40 & 40 & 40 & $11.80 \%$ \\
\hline PUNTAJE MÁXIMO & & & 340 & 340 & 339.2 & $99.70 \%$ \\
\hline
\end{tabular}

Realizado por: Los autores.

En relación al puntaje máximo que es 340 se puede evidenciar que la Metodología Diagnóstica tiene un cumplimiento en 339,2 que corresponde al $99.7 \%$ del total de historias analizadas, siendo un parámetro de evaluación satisfactoria en los índices de calidad.

Tabla 8. Contenido Notas de Evolución en la Historia Clínica.

\begin{tabular}{lcccccc}
\hline NOTAS DE EVOLUCION & AUS & MED & MAX & $\begin{array}{c}\text { PUNTAJE } \\
\text { IDEAL }\end{array}$ & $\begin{array}{c}\text { PUNTAJE } \\
\text { ALCANZADO }\end{array}$ & $\%$ \\
\hline $\begin{array}{l}\text { Se anota, interpreta } \\
\text { resultados de exámenes } \\
\text { auxiliares. }\end{array}$ & 0 & 40 & 80 & 80 & 1.6 & $0.80 \%$ \\
$\begin{array}{l}\text { Se anota interconsulta e } \\
\text { indicaciones del médico } \\
\text { consultante }\end{array}$ & 0 & 0 & 20 & 20 & 0 & $0.00 \%$ \\
$\begin{array}{l}\text { Se anota si el paciente están } \\
\text { de alta, será referido o } \\
\text { contra referido }\end{array}$ & 0 & 0 & 20 & 20 & 0.8 & $0.40 \%$ \\
$\begin{array}{l}\text { Evolución de acuerdo al } \\
\text { SOAP }\end{array}$ & 0 & 30 & 60 & 60 & 0 & $0.00 \%$ \\
$\begin{array}{l}\text { Aparece nombre y firma } \\
\text { del médico que hizo la nota } \\
\text { de evolución }\end{array}$ & 0 & 10 & 20 & 20 & 0 & $0.00 \%$ \\
\hline \begin{tabular}{l} 
PUNTAJE MÁxIMO \\
\hline
\end{tabular} & & & $\mathbf{2 0 0}$ & $\mathbf{2 0 0}$ & $\mathbf{2 . 4}$ & $\mathbf{1 . 2 0 \%}$ \\
\hline
\end{tabular}

Realizado por: Los autores. 
En relación al puntaje máximo que es 200, se puede evidenciar que las Notas de Evolución en la historia clínica registradas corresponden a 2.4, lo cual pertenece al $1.2 \%$, siendo este el parámetro de mayor incumplimiento en el llenado correcto de la historia clínica.

Tabla 9. Contenido Tratamiento y Órdenes del Médico en la Historia Clínica.

\begin{tabular}{|c|c|c|c|c|c|c|}
\hline $\begin{array}{c}\text { TRATAMIENTO Y } \\
\text { ORDENES DEL MEDICO }\end{array}$ & AUS & MED & MAX & $\begin{array}{l}\text { PUNTAJE } \\
\text { IDEAL }\end{array}$ & $\begin{array}{c}\text { PUNTAJE } \\
\text { ALCANZADO }\end{array}$ & $\%$ \\
\hline $\begin{array}{l}\text { Tratamiento médico o } \\
\text { procedimiento de cirugía } \\
\text { menor fueron apropiados } \\
\text { de acuerdo a protocolos. }\end{array}$ & 0 & 40 & 80 & 80 & 80 & $36.40 \%$ \\
\hline $\begin{array}{l}\text { Se anota medicamentos } \\
\text { dosis, vía de } \\
\text { administración, } \\
\text { periodicidad y duración. }\end{array}$ & 0 & $20 / 40$ & 60 & 60 & 60 & $27.30 \%$ \\
\hline $\begin{array}{l}\text { Se anota el tipo de dieta de } \\
\text { acuerdo a patología cuando } \\
\text { es pertinente. }\end{array}$ & 0 & 0 & 20 & 20 & 20 & $9.10 \%$ \\
\hline $\begin{array}{l}\text { Presencia o ausencia de } \\
\text { complicaciones. }\end{array}$ & 0 & 0 & 60 & 0 & 0 & $0.00 \%$ \\
\hline PUNTAJE MÁXIMO & & & 220 & 160 & 160 & $72.80 \%$ \\
\hline
\end{tabular}

En relación al puntaje máximo que es 220 , se puede evidenciar que el registro del Tratamiento y Órdenes del Médico en la Historia alcanza un total de 160, siendo el parámetro que no se cumple el detallar si existieron o no complicaciones.

Tabla 10. Contenido tratamiento y órdenes del médico en la historia clínica.

\begin{tabular}{|c|c|c|c|c|c|c|}
\hline EVALUACION FINAL Y OBSERVACIONES & AUS & MED & MAX & $\begin{array}{l}\text { PUNTAJE } \\
\text { IDEAL }\end{array}$ & $\begin{array}{l}\text { PUNTAJE } \\
\text { ALCANZA } \\
\text { DO }\end{array}$ & $\%$ \\
\hline $\begin{array}{l}\text { Los documentos de la historia clínica aparecen } \\
\text { en el orden establecido por las normas } \\
\text { institucionales. }\end{array}$ & 0 & 10 & 20 & 20 & 20 & $20.00 \%$ \\
\hline $\begin{array}{l}\text { En caso de enfermedades de vigilancia } \\
\text { Epidemiológica, obligatoria se comunicó } \\
\text { oportunamente a epidemiología }\end{array}$ & 0 & 0 & 10 & 10 & 10 & $10.00 \%$ \\
\hline Se consignó código alfanumérico (CIE-10) & 0 & 0 & 20 & 20 & 9.3 & $9.30 \%$ \\
\hline Uso de formatos oficiales en la historia clínica & 0 & 10 & 20 & 20 & 20 & $20.00 \%$ \\
\hline $\begin{array}{l}\text { En contenido de la historia clínica es útil para la } \\
\text { enseñanza e investigación }\end{array}$ & & 0 & 30 & 30 & 28.95 & $28.95 \%$ \\
\hline PUNTAJE MÁXIMO & & & 100 & 100 & 88.25 & $88.25 \%$ \\
\hline
\end{tabular}

Realizado por: Los autores

En relación al puntaje máximo que es 100, se puede evidenciar que el registro Evaluación Final y Observación en la Historia indica 88.25, lo cual corresponde al 88.25\%, siendo el parámetro de diagnóstico CIE 10 el que no se anota de manera correcta, e incluso no es llenado. 
Tabla 11. Revisión y comparación de las historias clínicas.

\begin{tabular}{|c|c|c|}
\hline $\begin{array}{c}\text { HISTORIAS } \\
\text { CLINICAS }\end{array}$ & $\begin{array}{l}\text { PUNTAJE } \\
\text { OBTENIDO EN LA } \\
\text { REVISION DE LA } \\
\text { HCL }\end{array}$ & $\begin{array}{l}\text { EQUIVALENTE } \\
\text { NUMERICO EN } \\
\text { PORRCENTAJE }\end{array}$ \\
\hline 76 & 700 & $70.00 \%$ \\
\hline 11 & 705 & $70.50 \%$ \\
\hline 7 & 730 & $73.00 \%$ \\
\hline 5 & 675 & $67.50 \%$ \\
\hline 1 & 685 & $68.50 \%$ \\
\hline 1 & 785 & $78.50 \%$ \\
\hline 1 & 695 & $69.50 \%$ \\
\hline 1 & 720 & $72.00 \%$ \\
\hline 4 & 690 & $69.00 \%$ \\
\hline 1 & 780 & $78.00 \%$ \\
\hline 1 & 750 & $75.00 \%$ \\
\hline 1 & 660 & $66.00 \%$ \\
\hline 100 & 3495 & $857.50 \%$ \\
\hline
\end{tabular}

De la revisión realizada en la muestra de 100 historias clínicas podemos evidenciar que: 11 historias clínicas alcanzaron puntaje entre $72 \%$ a $78 \%$ (equivalente numérico en porcentaje), que corresponde a BUENO, 89 historias clínicas alcanzaron puntaje entre $66 \%$ a $70 \%$ (equivalente numérico en porcentaje) que corresponde a MALO. 


\section{Conclusiones.}

- El análisis de la calidad asistencial de acuerdo a los indicadores obtenidos de 100 historias clínicas de consulta externa de primer nivel, en el Centro de Salud "A" FM MAS muestra que el $11 \%\left(\mathrm{~N}^{\circ} 11\right)$ de las historias clínicas alcanzaron un puntaje equivalente a BUENO (72\% a 78\%), y que el 89\% ( $\left.\mathrm{N}^{\circ} 89\right)$ de historias clínicas alcanzaron un puntaje equivalente a MALO (66\% a 70\%).

- Se determinó que el parámetro que representa mayor falla en el llenado de una historia clínica de buena calidad en consulta externa del C.S "A" FM MAS es Contenido Notas de Evolución en la Historia Clínica, alcanzando apenas un puntaje $1.2 \%$.

- Se estableció que el parámetro que representa menor falla en el llenado de una historia clínica de buena calidad en consulta externa del C.S "A" FM MAS es Contenido de Metodología Diagnóstica en la Historia Clínica, alcanzando un puntaje de $99.7 \%$.

- Se debe socializar los resultados obtenidos en este estudio y posteriormente capacitar al personal médico y de enfermería en el correcto registro y llenado de historias clínicas, así como a planilla dores y facturadores.

- Se debe mejorar la calidad del llenado de historias clínicas para evitar objeciones durante la auditoria de calidad por parte del Subsistema de Salud ISSFA, y su posterior cancelación de valores correspondientes a atenciones médicas.

\section{Referencias bibliográficas.}

1.- Díaz, R., Sánchez, T., Díaz, N., \& Hernández, S. Manual de Calidad Asistencial. Edita: SESCAM Servicio de Salud de Castilla-La Mancha. (2009). Disponible en http://sescam.castillalamancha.es/sites/sescam.castillalamancha.es/files/documentos/p df/20131017/manual_calidad_asistencial_de_la_seca.pdf.

2.-Cordero, F., \& Vergara, Constitución del Ecuador. (2008). Disponible en: www.oas.org/jurídico/mal/spa/ecu/sp_ecu-int-text-const.pdf

3.- Lucio R., Villares N., \& Enríquez, R. Sistema de Salud de Ecuador. Salud Pública México. 2019; Vol. 53:180-185. Disponible en: http://www.scielo.org.mx/scielo.php?script=sci_arttext\&pid=S0036-6342011000800013

4.- Vance, C., Serrano, J., Ponce, J., \& Guijarro, F. Convenio Marco. Dirección Nacional de Articulación de la Red Pública y Complementaria de Salud. Disponible en: http://instituciones.msp.gob.ec/images/Documentos/subse_gobernanza/Conven io_RP S\%202015.pdf

5.-WHO Working Group. The principles of quality assurance. Qual Assur Health Care.

Pudmed. 2019:79-95. Diponible en:

www.ncbi.nlm.nih.gov/pubmed/2490962 
6.- Alcaraz, M., Napóles, y, Chaveco, I., \& Martínez, M. La historia clínica: un documento básico para el personal médico. MEDISAN. 2019 Disponible en: http://scielo.sld.cu/scielo.php?script=sci_arttext\&pid=S1029-3019201000070001

7.- Muniagurria, A. Algunas apreciaciones sobre el Método Clínico. 2019 Disponible en: http://www.villavicencio.org.ar/pdf/023 Algunas apreciaciones sobre el Método Clinico.pdf

8.- Aguado, J. A., Gastón, J. L., Bueno, A., \& López, R. Estudio de la Calidad de los Registros en un Centro de Atención Primaria. La Gaceta Sanitaria. 2019 Disponible en http://www.gacetasanitaria.org/es-estudio-la-calidad-los-registros-articuloS021391119171073X.

9.- Pavón, L., \& García, M. La auditoría Médica como Herramienta de Control en la Administración Hospitalaria en el Ecuador. Vol 1. 1er ed. Quito Editor. 2017. 


\section{PARA CITAR EL ARTÍCULO INDEXADO.}

Zabala Chico, M., Arévalo Méndez, V., \& Cáceres Manzano, P. (2019). Análisis de indicadores de la calidad asistencial sanitaria del Centro de Salud "A" Fuerte Militar Marco Aurelio Subía, en el periodo comprendido entre julio y diciembre del 2018. Ciencia Digital, 3(2.1), 181-191. https://doi.org/10.33262/cienciadigital.v3i2.1.438

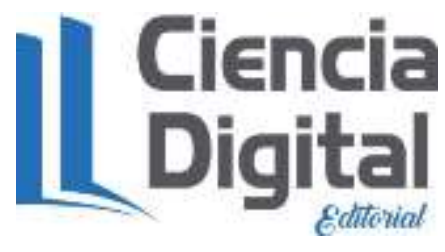

El artículo que se publica es de exclusiva responsabilidad de los autores y no necesariamente reflejan el pensamiento de la Revista Ciencia Digital.

El artículo queda en propiedad de la revista y, por tanto, su publicación parcial y/o total en otro medio tiene que ser autorizado por el director de la Revista Ciencia Digital.
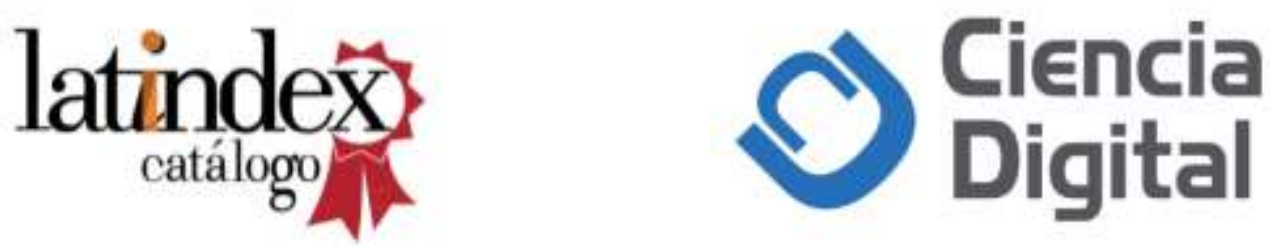